\title{
ON THE DIRECTIONAL DEPENDENCE AND NULL SPACE FREEDOM IN UNCERTAINTY BOUND IDENTIFICATION
}

\author{
K.B. Lim, ${ }^{*} \quad$ D.P. Giesy ${ }^{\dagger}$ \\ NASA Langley Research Center \\ Hampton, Virginia
}

\begin{abstract}
In previous work, the determination of uncertainty models via minimum norm model validation is based on a single set of input and output measurement data. Since uncertainty bounds at each frequency is directionally dependent for multivariable systems, this will lead to optimistic uncertainty levels. In addition, the design freedom in the uncertainty model has not been utilized to further reduce uncertainty levels. The above issues are addressed by formulating a minmax problem. An analytical solution to the min-max problem is given to within a generalized eigenvalue problem, thus avoiding a direct numerical approach. This result will lead to less conservative and more realistic uncertainty models for use in robust control.
\end{abstract}

\section{Introduction}

In applying multivariable robust control analysis and synthesis techniques to linear, time-invariant systems, as in for example [1], a set of plants as defined by a nominal and uncertainty model are required a priori. Nominal models are usually associated with a single "best" model, although what is considered "best" is debatable. Mathematical models derived from first principles are typically used as nominal models or sometimes identified from system identification experiments. In some cases where the physical conditions are not accurately or reliably known due to causes unknown or when simple models are desirable, it makes sense to require that the set of plants in question at least satisfy model validation conditions [2]-[6] with respect to available input and output measurement data.

A recently proposed approach $[4,5,6]$ to obtaining uncertainty models from measurement data is based on searching for the smallest uncertainty set

*Aerospace Technologist, Guidance \& Control Branch, Flight Dynamics \& Control Division, k.b.lim@larc.nasa.gov

†Aerospace Technologist, Guidance \& Control Branch, Flight Dynamics \& Control Division, d.p.giesy@larc.nasa.gov that will validate all available data. The approach assumes a single set of input and output measurement data. For multivariable systems, this results in a particular input direction at each discrete frequency which in turn leads to optimistic uncertainty bounds. In addition, the need to admit model validating solutions implys a sufficient degree of freedom in uncertainty structure selection. This remaining freedom in a certain null space has not been utilized to reduce the minimum norm uncertainty bounds. In this paper, we investigate both issues. The problem formulation leads to a min-max problem and the conditions for a solution is given.

We begin by first considering the standard $P \Leftrightarrow \Delta$ system shown in figure 1 . Let the overall structured uncertainty be defined by the block diagonal matrices

$$
\Delta=\operatorname{diag}\left(\Delta^{1}, \ldots, \Delta^{\tau}\right) ; \quad \Delta^{i} \in C^{m_{i} \times n_{i}}, i=1, \ldots, \tau(1)
$$

and the set of all block diagonal and stable, rational transfer function matrices be given by

$$
\mathcal{D}=\left\{\Delta(\cdot) \in R H_{\infty}: \Delta^{i}\left(s_{0}\right) \in C^{m_{i} \times n_{i}}, \forall s_{0} \in \bar{C}_{+}\right\}(2)
$$

where $\tau$ and $\bar{C}_{+}$denote the number of uncertainty blocks and the closed right-half plane, respectively [7]. The output error is given by

$$
e_{y}:=y \Leftrightarrow \tilde{y}=y \Leftrightarrow \mathcal{F}_{u}(P, \Delta) u
$$

where the output, input, and upper linear fractional transformation of $(P, \Delta)$, are denoted by $y, u$, and $\mathcal{F}_{u}(P, \Delta)$, and are variables in the $\mathrm{z}$-domain so that they are complex vectors containing both gain and phase information.

For model validation $[4,5,6]$ with respect to input and output data $u$ and $y$, the output error is set to zero so that from (3)

$$
y \Leftrightarrow P_{22} u=P_{21} \Delta\left(I \Leftrightarrow P_{11} \Delta\right)^{-1} P_{12} u
$$

Observe from the left hand side of (4) that if the given nominal model $P_{22}$ and input cancels the measured outputs (to within white noise), then $\Delta=0$ validates the model. This is the case where a single 


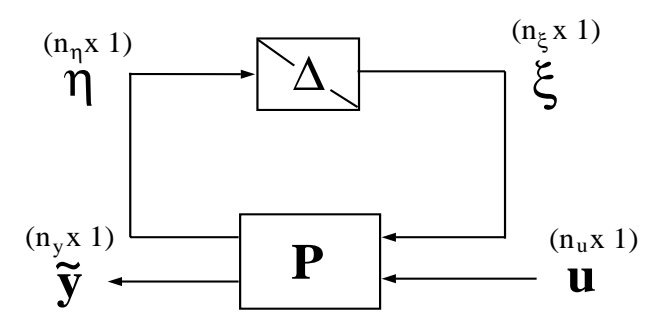

Figure 1: $P \Leftrightarrow \Delta$ structure in standard form.

"optimal" model can be found such that the output errors are perfectly uncorrelated to the inputs. In this ideal case, a single "true" model is available and robustness issues with respect to model error do not exist. Otherwise, note that the nominal model error is directionally dependent on the inputs. Hence, with a different (directional) choice of inputs, the mininum norm model validating uncertainty computed will also be different.

\section{Problem Formulation}

To address the directional dependence of the mininum norm model validating uncertainty with respect to the test input, we assume that $n_{u}$ sets of inputs and corresponding outputs at each frequency are available and are arranged in the matrix forms

$$
U:=\left[u_{1}, \ldots, u_{n_{u}}\right] ; \quad Y:=\left[y_{1}, \ldots, y_{n_{u}}\right]
$$

These sets of inputs and outputs can be formed from segments of a long identification data or independent identification experiments. Assuming a linear timeinvariance system, a general input

$$
u=U \alpha
$$

will result in output

$$
y=Y \alpha
$$

so that the error equation for a general input signal is

$$
e_{y}(\alpha, \Delta)=Y \alpha \Leftrightarrow \mathcal{F}_{u}(P, \Delta) U \alpha
$$

Note that $\alpha$ is a complex coefficient vector which help to span the $n_{u}$ dimensional vector space. The approach for handling the directional dependence is to search for the largest possible mininum norm model validating uncertainty over all possible test inputs.

Consider model validating a linear combination of input/output data set, i.e.,

$$
e_{y}(\alpha, \Delta)=0 ; \quad \forall \alpha
$$

From (8), this condition is

$$
P_{21} \Delta\left(I \Leftrightarrow P_{11} \Delta\right)^{-1} P_{12} U \alpha=\left(Y \Leftrightarrow P_{22} U\right) \alpha ; \quad \forall \alpha(10)
$$

Using the earlier approach and making the assumptions as given in $[4,5]$, the $\Delta$ can be unscrambled to the form

$$
\Delta x=y ; \quad \forall \alpha
$$

where

$$
\begin{aligned}
x(\alpha, \phi) & :=B \alpha+P_{11} \phi \\
y(\alpha, \phi) & :=A \alpha+\phi
\end{aligned}
$$

and

$$
\begin{aligned}
& A=P_{21}^{+}\left(Y \Leftrightarrow P_{22} U\right) \\
& B=P_{12} U+P_{11} P_{21}^{+}\left(Y \Leftrightarrow P_{22} U\right)
\end{aligned}
$$

$\phi \in \mathcal{N}\left(P_{21}\right)$, where $\mathcal{N}(\cdot)$ denotes the null space. The variables depend on the arguments, $\alpha$ and $\phi$, and in the remaining development, these arguments will not be explicitly used for simplicity.

To obtain lower bounds on the uncertainty components, partition in a conformal manner with respect to the uncertainty blocks the following:

$$
x=\operatorname{col}\left(x^{1}, \ldots, x^{\tau}\right) ; \quad y=\operatorname{col}\left(y^{1}, \ldots, y^{\tau}\right)
$$

Due to the block diagonality of $\Delta$,

$$
\Delta x=y, \forall \alpha \quad \Leftrightarrow \quad \Delta^{i} x^{i}=y^{i}, \forall(i, \alpha)
$$

and in addition

$$
\bar{\sigma}(\Delta)=\max _{i} \bar{\sigma}\left(\Delta^{i}\right), \quad \forall \alpha
$$

Consider the following Lemmas as given in $[4,5]$.

\section{Lemma 1:}

For each component, $x^{i} \neq 0$,

$$
\bar{\sigma}\left(\Delta^{i}\right)=\left\|\Delta^{i}\right\|_{2} \geq \frac{\left\|y^{i}\right\|_{2}}{\left\|x^{i}\right\|_{2}}, \quad \forall(i, \alpha) .
$$

Lemma 2:

Let $y^{i} \in C^{m_{i}}, x^{i} \in C^{n_{i}}, x^{i} \neq 0, \exists \Delta^{i} \in C^{m_{i} \times n_{i}}$ : $\Delta^{i} x^{i}=y^{i}, \quad \bar{\sigma}\left(\Delta^{i}\right)=\frac{\left\|y^{i}\right\|_{2}}{\left\|x^{i}\right\|_{2}}$.

The first lemma gives a lower bound on the maximum singular value for the $i$ th block diagonal uncertainty and the input/output data defined by $\alpha$. In order to model validate the input/output relationship as defined by (17) for a given input vector function, $x^{i}$, and an output vector function, $y^{i}$, the smallest normed uncertainty is given by the lower bound in Lemma 1. Of course if there are several input/output data sets (corresponding to different $\alpha$ 's) to model validate, then the largest lower bound with respect to $\alpha$ is needed to validate all data given. The second lemma 
shows that the $i$ th component uncertainty, $\Delta^{i}$, can be chosen to achieve this lower bound. Thus Lemma 2 ensures that a minimum normed uncertainty exists which will validate the given data. Again, if there are several input/output data sets the largest bound with respect to $\alpha$ is necessary.

The problem is summarized in the following minmax problem:

$$
\text { Problem 1: } \max _{\alpha}\left(\min _{\phi \in \mathcal{N}\left(P_{12}\right)} J(\alpha, \phi):=\frac{\|y(\alpha, \phi)\|_{2}}{\|x(\alpha, \phi)\|_{2}}\right)
$$

The physical interpretations for $\alpha$ and $\phi$ are clear. The variable $\alpha$ represents all possible input disturbances in the system identification experiment whereas the variable $\phi$ denotes the remaining freedom in the assumed uncertainty. Obviously, a larger number of uncertainty components in $\Delta$ will generally give greater freedom in $\phi$ as realized by an increased dimension of the null space, $\mathcal{N}$.

\section{Solutions}

\subsection{General Case}

In this section we consider the general min-max problem defined in Problem 1. To avoid dealing with square roots, we consider the equivalent min-max of the ratio of norms squared. The problem considered is given as follows:

$$
\max _{\alpha}\left(\min _{\psi} J(\alpha, \phi(\psi)):=\frac{\|A \alpha+N \psi\|_{2}^{2}}{\|B \alpha+D \psi\|_{2}^{2}}\right)
$$

where

$$
\begin{aligned}
N^{H} A & =0 \\
N^{H} N & =I \\
D & =P_{11} N
\end{aligned}
$$

The matrix $N$ denotes an orthogonal basis for the null space $\mathcal{N}$ and (22) shows its orthogonality with respect to the range space of the pseudo-inverse $P_{21}^{+}$. The free parameter $\psi$ has the same dimension as the null space and is related to $\phi$ by

$$
\begin{aligned}
& \phi= N \psi \\
& \text { Let } \\
& A:=A_{r}+j A_{i} \quad \in C^{r \times n} \\
& N:=N_{r}+j N_{i} \in C^{r \times m} \\
& B:=B_{r}+j B_{i} \in C^{s \times n} \\
& D:=D_{r}+j D_{i} \in C^{s \times m} \\
& \alpha:=\alpha_{r}+j \alpha_{i} \in C^{n \times 1} \\
& \psi:=\psi_{r}+j \psi_{i} \in C^{m \times 1}
\end{aligned}
$$

It can be shown that

$$
\begin{aligned}
& \|A \alpha+N \psi\|_{2}^{2}=\xi^{T} \tilde{P}^{T} \tilde{P} \xi:=a(\alpha, \psi) \geq 0 \\
& \|B \alpha+D \psi\|_{2}^{2}=\xi^{T} \tilde{Q}^{T} \tilde{Q} \xi:=b(\alpha, \psi) \geq 0
\end{aligned}
$$

where the real column vector variable is

$$
\xi:=\left\{\begin{array}{c}
\alpha_{r} \\
\alpha_{i} \\
\psi_{r} \\
\psi_{i}
\end{array}\right\}
$$

and

$$
\begin{aligned}
& \tilde{P}=\left[\begin{array}{ll}
\tilde{P}_{1} & \tilde{P}_{2}
\end{array}\right]=\left[\begin{array}{cccc}
A_{r} & \Leftrightarrow A_{i} & N_{r} & \Leftrightarrow N_{i} \\
A_{i} & A_{r} & N_{i} & N_{r}
\end{array}\right] \\
& \tilde{Q}=\left[\begin{array}{ll}
\tilde{Q}_{1} & \tilde{Q}_{2}
\end{array}\right]=\left[\begin{array}{cccc}
B_{r} & \Leftrightarrow B_{i} & D_{r} & \Leftrightarrow D_{i} \\
B_{i} & B_{r} & D_{i} & D_{r}
\end{array}\right]
\end{aligned}
$$

The positive definiteness of $\tilde{Q}^{T} \tilde{Q}$ is assumed from a physical argument. Observe that if $b=0$ and $a \neq 0$, then $J$, the lower bound on the norm of model validating uncertainty is unbounded, which is to say a model validating uncertainty do not exist. Hence we assume that $b>0 \forall \xi \neq 0$ so that $\tilde{Q}^{T} \tilde{Q}>0$ or equivalently, $\tilde{Q}$ has full column rank. The cost function is then written as

$$
J(\alpha, \psi)=\frac{a(\alpha, \psi)}{b(\alpha, \psi)} \geq 0
$$

As figure 2 illustrates, a solution to the min-max problem lies at a saddle point. The particular type of saddle point we seek must possess the following properties: it should be stationary with respect to $\xi$, local maximum with respect to $\alpha$, and local minimum with respect to $\psi$. Specifically,

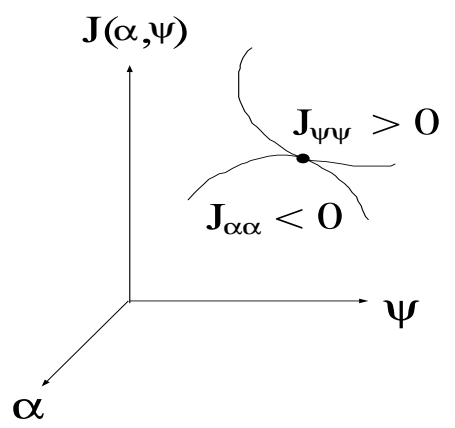

Figure 2: Geometry of a min-max solution: saddle point.

$\frac{\partial J}{\partial \xi^{T}}=0 \Leftrightarrow \frac{\partial J}{\partial \alpha^{T}}=0, \frac{\partial J}{\partial \psi^{T}}=0 \quad$ stationarity $(39)$ 
and

$$
\begin{array}{lll}
\frac{\partial}{\partial \alpha}\left(\frac{\partial J}{\partial \alpha^{T}}\right)<0 & \text { local maximum wrt } \alpha \\
\frac{\partial}{\partial \psi}\left(\frac{\partial J}{\partial \psi^{T}}\right)>0 & \text { local minimum wrt } \psi
\end{array}
$$

By taking partial derivatives, it can be shown that the stationary condition reduce to

$$
\left[\tilde{P}^{T} \tilde{P} \Leftrightarrow J \tilde{Q}^{T} \tilde{Q}\right] \xi=0
$$

It can also be shown that the local maximum and minimum conditions reduce to the sign definiteness conditions

$$
\begin{aligned}
& \tilde{P}_{1}^{T} \tilde{P}_{1} \Leftrightarrow J \tilde{Q}_{1}^{T} \tilde{Q}_{1}<0 \\
& \tilde{P}_{2}^{T} \tilde{P}_{2} \Leftrightarrow J \tilde{Q}_{2}^{T} \tilde{Q}_{2}>0
\end{aligned}
$$

Finally, it follows that the above negative and positive definiteness conditions in (43) and (44) respectively, is equivalent to the condition

$$
\bar{\sigma}\left(\tilde{P}_{1} L_{1}^{-T}\right)<J<\underline{\sigma}\left(\tilde{P}_{2} L_{2}^{-T}\right)
$$

where $L_{i}$ are square, nonsingular matrices derived from the singular value decompositions of $\tilde{Q}_{i}$ [8], i.e,

$$
\tilde{Q}_{i}=U_{i} \Sigma_{i} V_{i}^{T} \quad L_{i}=V_{i} \Sigma_{i}, \quad i=1,2 .
$$

Note that $\tilde{Q}_{1}$, and $\tilde{Q}_{2}$ will have full column rank matrices since $\tilde{Q}$ was earlier assumed to have full column rank on physical grounds. To recapitulate, the min-max problem defined by Problem 1 reduces to finding $\alpha$ and $\psi$, such that $J$ is stationary with respect to both $\alpha$ and $\psi$, i.e., the equality (42) holds, and the curvature of $J$ with respect to $\alpha$ is negative and is positive with respect to $\psi$, i.e., the inequality (45) holds.

To satisfy the conditions defined in (42) and (45), first note that both $J$ and $\xi$ are unknowns in (42) so that this equation can be treated as a real, symmetric generalized eigenvalue problem. The functional dependence of both $a$ and $b$ and hence $J$ on $\xi$ is not constraining as evident from premultiplying (42) by $\xi^{T}$ and solving for $J$. Hence a simple and direct solution algorithm follows. First, find all stationary points by solving for all the eigenvalues and corresponding eigenvectors for the eigenvalue problem in (42)

$$
J^{k}:=\left(\frac{a}{b}\right)_{k}, \quad \xi^{k}=\left\{\begin{array}{c}
\alpha_{r}^{k} \\
\alpha_{i}^{k} \\
\psi_{r}^{k} \\
\psi_{i}^{k}
\end{array}\right\},
$$

where $k=1, \ldots, 2\left(n_{u}+\operatorname{dim}(\mathcal{N})\right)$. Second, check to see if any of the $\lambda_{k}$ 's lie within the inequality bound (45). The results are summarized in the following:
Proposition 1:

Let $J^{k}, \xi^{k}$ be the eigenpair of (42). Then the eigenpair is a solution to the min-max problem in (20) only if $J^{k}$ satisfy the inequalities (45).

If more than one eigenvalue lie within the inequality bound given in (45), this indicates that there is more than one saddle point and the smallest eigenvalue should be chosen since the square root of these eigenvalues directly correspond to uncertainty lower bounds. From a computational standpoint, the real, symmetric generalized eigenvalue problem that needs to be solved is of dimension $2\left(n_{u}+\operatorname{dim}(\mathcal{N})\right)$, which will not likely be a problem for most practical applications.

\subsection{Special Case, $\phi=0$}

Consider the special case of $\phi=0$ or when $\mathcal{N}\left(P_{21}\right)=$ $\{0\}$. The mini-max problem reduces to the single optimization problem:

$$
\text { Problem } 2: \max _{\alpha} J(\alpha, 0):=\frac{\|y(\alpha, 0)\|_{2}}{\|x(\alpha, 0)\|_{2}}=\frac{\|A \alpha\|_{2}}{\|B \alpha\|_{2}}(48)
$$

Since $\Delta$ must be finite in satisfying the model validation condition (9) for all nonzero $\alpha, B^{H} B$ must be positive definite. With the nonsingular (and hence unique) coordinate transformation

$$
L^{T} \alpha=\beta
$$

the optimization problem reduces to

$$
\begin{aligned}
\max _{\alpha} J(\alpha, 0) & =\max _{\alpha} \frac{\|A \alpha\|_{2}}{\|B \alpha\|_{2}} \\
& =\max _{\beta} \frac{\left\|A L^{-T} \beta\right\|_{2}}{\|\beta\|_{2}} \\
& =\bar{\sigma}\left(A L^{-T}\right)
\end{aligned}
$$

The above development is summarized in the following.

\section{Proposition 2:}

The largest lower bound of the uncertainty with respect to all possible linear combinations of a set of $n_{u}$ input/output data is given as

$$
\bar{\sigma}(\Delta) \geq J(\bar{\alpha}, 0)=\bar{\sigma}\left(A L^{-T}\right) .
$$

Consider the special case where a specific lower bound is used instead of any linear combination. For example, let $\delta_{j}$ denote the $j$ th column of an $n_{u}$ by $n_{u}$ identify matrix. Then the $j$ th input vector corresponds to $\alpha=\delta_{j}$ so that 


$$
u_{j}=U \delta_{j} ; \quad y_{j}=Y \delta_{j}
$$

From (49) the corresponding $\beta$ is

$$
\beta_{j}=L^{T} \delta_{j}
$$

so that from Proposition 2

$$
\begin{aligned}
\frac{\left\|y\left(\delta_{j}, 0\right)\right\|_{2}}{\left\|x\left(\delta_{j}, 0\right)\right\|_{2}} & =\frac{\left\|A L^{-T} \beta_{j}\right\|_{2}}{\left\|\beta_{j}\right\|_{2}} \leq \bar{\sigma}\left(A L^{-T}\right) \leq \bar{\sigma}(\Delta) ; \\
& \forall \quad j=1, \ldots, n_{u}
\end{aligned}
$$

The expression on the left hand side of (56) denotes an uncertainty bound based on a single input/output data and is given in the earler works $[4,5]$.

\subsection{Structured Uncertainty}

To compute norm bounds for unstructured uncertainty, the maximum singular value in (53) or the eigenvalues in (47) need to be computed for general and special case respectively. However, for structured uncertainty case, eigenvector $\xi^{k}$ and the maximizing principal vector $\bar{\beta}$ is needed to compute individual component uncertainty bounds for the general and special case respectively.

For the special case, the norm bound on the $i$ th component uncertainty is given from (19) as

$$
\bar{\sigma}\left(\Delta^{i}\right) \geq \frac{\left\|\left[A L^{-T} \bar{\beta}\right]^{i}\right\|_{2}}{\left\|\bar{\beta}^{i}\right\|_{2}}
$$

The superscript $i$ in the right hand side of (57) denotes the $i$ th component of the column vectors. The principal vector, $\bar{\beta}$, comes from the singular value decomposition

$$
A L^{-T}=P \cdot \operatorname{diag}\left(\bar{\sigma}\left(A L^{-T}\right), \ldots\right) \cdot[\bar{\beta}, \ldots]^{T}
$$

Similarly, for the general case, the norm bound on the $i$ th component uncertainty is also given from (19) as

$$
\bar{\sigma}\left(\Delta^{i}\right) \geq \frac{\left\|y^{i}\left(\alpha^{k}, \phi\left(\psi^{k}\right)\right)\right\|_{2}}{\left\|x^{i}\left(\alpha^{k}, \phi\left(\psi^{k}\right)\right)\right\|_{2}}
$$

where the subscript $k$ in (59) denotes the smallest eigenpair solution that satisfies the min-max inequality bounds.

\section{Conclusion}

The issue of directional dependence of multivariable uncertainty models is addressed along with the uncertainty design freedom beyond model validation. The problem is formulated as a search for a saddle point in a finite dimensional, complex space. Numerical and experimental validation and applications of the proposed solution is underway. It is expected that these results will lead to less conservative and more realistic uncertainty models for use in robust control.

\section{References}

[1] Balas, G.J., Doyle, J.C., Glover, K., Packard, A., and Smith, R., $\mu$-Analysis and Synthesis Toolbox, MUSYN Inc., Minneapolis, MN, 1994.

[2] Smith, R.S., Doyle, J.C., "Model Validation: A connection between robust control and identification," IEEE Transactions on Automatic Control, vol 37, No 7, July 1992, pp.942-952.

[3] Kumar, A., and Balas, G.J., "A scaling approach to model validation in the $\mu$ framework," 1995 American Control Conference, pp.693-697.

[4] Lim, K.B., Balas, G.J., and Anthony, T.C., "Minimum-norm model validating identification for robust control," AIAA Paper No. 96-3717.

[5] Lim, K.B., "Closed form solution for minimum norm model validating uncertainty," 1997 American Control Conference, Paper No. 97050.

[6] Lim, K.B., Cox, D.E., Balas, G.J., and Juang, J-N., "Validation of an experimentally derived uncertainty model," AIAA Paper No. 97-0244.

[7] Stein, G., and Doyle, J.C., "Beyond singular values and loop shapes," Journal of Guidance, Control, and Dynamics, vol.14, No.1, Jan-Feb 91, pp.5-16.

[8] Golub, G.H., and Van Loan, C.F., Matrix Computations, The johns Hopkins University Press, 1983 , p.89. 